\title{
Electron Beam Expansion by Target Heating
}

\author{
Manuel Garcia
}

July 2, 1998

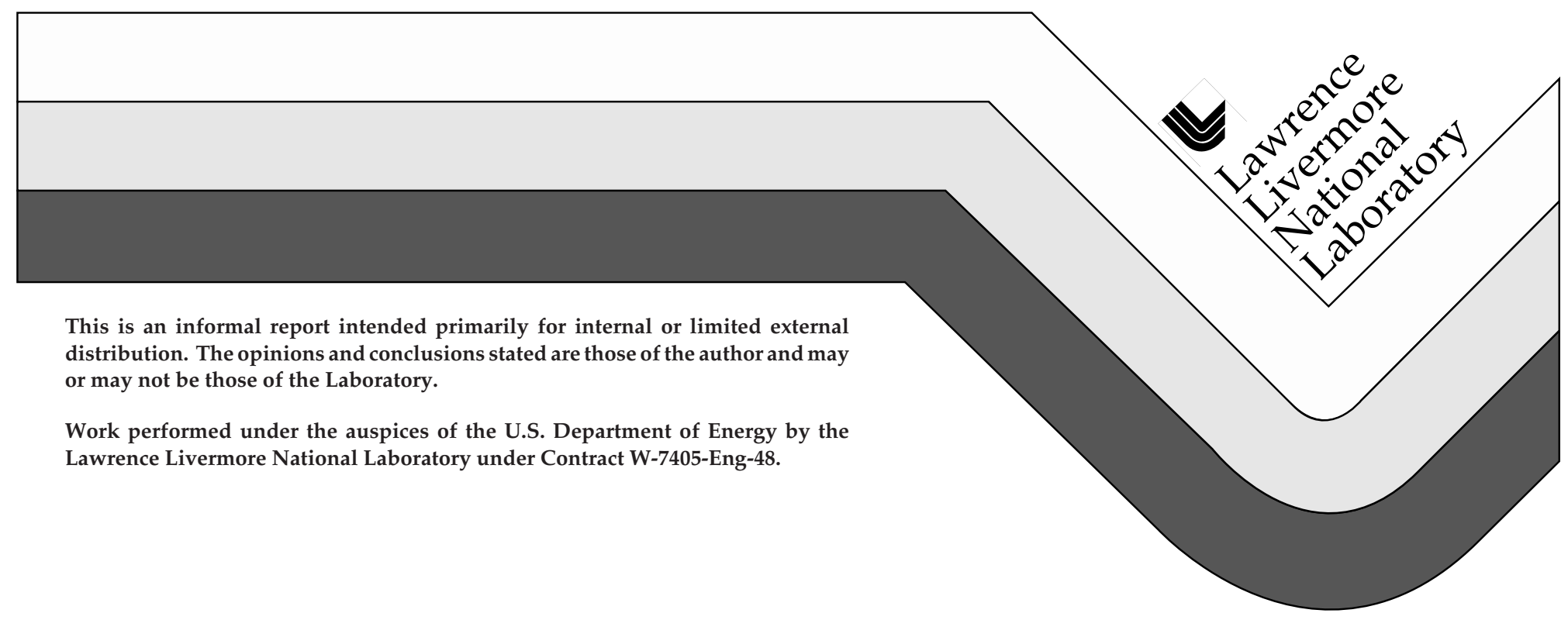




\section{DISCLAIMER}

This document was prepared as an account of work sponsored by an agency of the United States Government. Neither the United States Government nor the University of California nor any of their employees, makes any warranty, express or implied, or assumes any legal liability or responsibility for the accuracy, completeness, or usefulness of any information, apparatus, product, or process disclosed, or represents that its use would not infringe privately owned rights. Reference herein to any specific commercial product, process, or service by trade name, trademark, manufacturer, or otherwise, does not necessarily constitute or imply its endorsement, recommendation, or favoring by the United States Government or the University of California. The views and opinions of authors expressed herein do not necessarily state or reflect those of the United States Government or the University of California, and shall not be used for advertising or product endorsement purposes.

This report has been reproduced directly from the best available copy.

Available to DOE and DOE contractors from the Office of Scientific and Technical Information P.O. Box 62, Oak Ridge, TN 37831

Prices available from (423) 576-8401

Available to the public from the National Technical Information Service

U.S. Department of Commerce 5285 Port Royal Rd., Springfield, VA 22161 


\title{
Electron beam expansion by target heating
}

\author{
Manuel Garcia \\ 2 July 1998
}

Lawrence Livermore National Laboratory, L-153, POB 808 Livermore, CA 94551-0808 USA

(925) 422- 6017, garcia22@1lnl.gov, FAX (925) 423-5080

An intense, pulsed, relativistic electron beam can heat a thin metal plate to a plasma state as it traverses. This rapid heating creates a large radial temperature gradient in the material. As heat flow is primarily by electron motion, currents arise because of the thermal gradient. This effect magnetizes the target material during the course of the beam. If this magnetization exceeds that of the vacuum field of the electron beam then it dominates beam dynamics within the material. Plasmas of interest easily cancel the space charge of the electron beam, so target magnetization would either add to the self-pinch of the electron beam, or cancel it and cause beam expansion. The analysis described here finds that target heating causes beam expansion.

A previous report described how an electron beam can heat, magnetize, and accelerate target material from a thin metal plate, see Reference 1 . In that analysis the electron beam was taken simply as a current density and pressure source in the equations of target material dynamics. Here we ask, how does the expanding, magnetized plasma affect the electron beam?

The governing equations of the material taken as an ideal fluid were derived in Reference 1 and are:

$$
\begin{aligned}
& \frac{\partial \rho}{\partial t}+\operatorname{div}(\rho \mathbf{v})=0, \\
& \rho\left(\frac{\partial \mathbf{v}}{\partial t}+\mathbf{v} \cdot \nabla \mathbf{v}\right)=-\nabla p+\operatorname{curl}\left(\frac{\mathbf{B}-\mathbf{B}_{0}}{\mu}\right) \times \mathbf{B}, \\
& \operatorname{div}(\mathbf{v} \times \mathbf{B})=\frac{-\rho_{0}}{\varepsilon}-\frac{\operatorname{curl}\left(\mathbf{B}-\mathbf{B}_{0}\right)}{\sigma \mu} \cdot \frac{\nabla \sigma}{\sigma},
\end{aligned}
$$




$$
\operatorname{curl}(\mathbf{v} \times \mathbf{B})=\frac{\partial \mathbf{B}}{\partial t}-\frac{\nabla^{2}\left(\mathbf{B}-\mathbf{B}_{0}\right)}{\sigma \mu}+\frac{\operatorname{curl}\left(\mathbf{B}-\mathbf{B}_{0}\right)}{\sigma \mu} \times \frac{\nabla \sigma}{\sigma} .
$$

Here $\mathbf{v}, \mathbf{B}, \rho$, and $p$ are the velocity, magnetic induction, density and pressure, respectively. An equation of state $p(\rho)$ completes the set. The conductivity is $\sigma$, and $\varepsilon$ and $\mu$ are the permittivity and permeability; $t$ is time, and $\rho_{0}$ is a source charge density. The current density of the electron beam is $\mathbf{j}_{0}=\operatorname{curl}\left(\mathrm{B}_{0} / \mu\right)$, where the source induction $\mathrm{B}_{0}$ is simply an alternate representation of the electron beam within the material. The current density in the material exclusive of the electron beam is $\mathbf{j}=\operatorname{curl}\left(\mathbf{B}-\mathrm{B}_{0}\right) / \mu$.

Hydrodynamic calculations (Reference 2) show that a $6 \mathrm{kA}, 20$ $\mathrm{MeV}, 60 \mathrm{~ns}$ FWHM, $1 \mathrm{~mm}$ diameter beam through a $1 \mathrm{~mm}$ thick tantalum plate will cause target material to bulge outward nearly 1 $\mathrm{mm}$ on each side of the plate by the end of the pulse, and to heat up to $2 \mathrm{eV}$ on the surface and $11 \mathrm{eV}$ in the interior. The material traversed by the electron beam has expanded by over 30\% during the course of the pulse. Even so, the density remains quite high and it is reasonable to consider this a steady, incompressible, neutral $\left(\rho_{0}=0\right)$ flow. Equations (1) - (4) now become:

$$
\begin{aligned}
& \operatorname{div}(\mathbf{v})=0 \\
& \rho(\mathbf{v} \cdot \nabla \mathbf{v})=-\nabla p+\mathbf{j} \times \mathbf{B}, \\
& \operatorname{div}(\mathbf{v} \times \mathbf{B})=-\frac{\mathbf{j}}{\sigma} \cdot \frac{\nabla \sigma}{\sigma}, \\
& \operatorname{curl}(\mathbf{v} \times \mathbf{B})=-\frac{\nabla^{2}\left(\mathbf{B}-\mathbf{B}_{0}\right)}{\sigma \mu}+\frac{\mathbf{j}}{\sigma} \times \frac{\nabla \sigma}{\sigma} .
\end{aligned}
$$

Because the plasma is dense, hot, and multiply ionized, the gradient of conductivity can be assumed parallel to that of temperature, and $\sigma \mu L^{2}$, the characteristic magnetic diffusion time for length scale $L$, is assumed large. Given this assumption, the terms to the right in equation (8) are seen to be comparable and small, and the system reduces to: 


$$
\begin{aligned}
& \operatorname{div}(\mathbf{v} \times \mathbf{B})=-\frac{\mathbf{j}}{\sigma} \cdot \frac{\nabla \sigma}{\sigma}, \\
& \mathbf{v} \times \mathbf{B}=\nabla \phi, \\
& \nabla\left(\frac{\rho V^{2}}{2}+p+\frac{B^{2}}{2 \mu}\right)=\frac{\mathbf{B} \cdot \nabla \mathbf{B}}{\mu}-\mathbf{j}_{0} \times \mathbf{B} .
\end{aligned}
$$

The nonuniform conductivity has the effect of producing a charge distribution, which in turn establishes an electrostatic field $-\nabla \phi$, and flow is a $-\nabla \phi \times \mathrm{B}$ drift. The energy density

$$
U=\frac{\rho v^{2}}{2}+p+\frac{B^{2}}{2 \mu}
$$

changes along the flow as a result of the curvature force of the magnetic induction and the body force introduced into the material by the electron beam.

Equation (10) also shows that the gradient of the energy density of the material, which is primarily thermal, exerts a body force on the electron beam $\mathbf{j}_{0}$. Assume that $\mathbf{B} \cdot \nabla \mathbf{B}=0$, for instance a purely azimuthal induction that only varies axially, then equation (10) is

$$
\nabla U=-\mathbf{j}_{0} \times \mathbf{B},
$$

which shows that $\mathbf{B}$ must be in the opposite sense to $\mathbf{B}_{0}$ for an energy density that increases toward the axis. The nonuniform energy density of the material forces the electron beam to expand. When the curvature force is absent, the velocity and magnetic induction are seen to be:

$$
\begin{aligned}
& \mathbf{v}=\frac{\mathbf{B} \times \nabla \phi}{B^{2}}, \\
& \mathbf{B}=\frac{\mathbf{j}_{0} \times \nabla U}{\mathbf{j}_{0}^{2}},
\end{aligned}
$$

and the electrostatic potential is given by equations (7) and (9) as 


$$
\nabla^{2} \phi=-\frac{\mathbf{j}}{\sigma} \cdot \frac{\nabla \sigma}{\sigma} .
$$

The velocity can be reduced to a more primitive form by eliminating B between equations (13) and (14),

$$
\mathbf{v}=-\mathbf{j}_{0} \frac{(\nabla \phi \cdot \nabla U)}{|\nabla U|^{2}}+\nabla U \frac{\left(\nabla \phi \cdot \mathbf{j}_{0}\right)}{|\nabla U|^{2}} \text {. }
$$

This shows velocity components aligned with the current source and the gradient of energy density. Figure 1 shows how the vector fields in the target plasma can be related when the velocity is outward. Heat pushes plasma away from the axis and generates currents that magnetize the target. When the gradient in energy density becomes sufficiently large, it can overwhelm the self-pinch force $\mathbf{j}_{0} \times \mathbf{B}_{0}$ of the electron beam and cause it to expand. An example in Reference 1 shows that $\mathbf{B}$ can grow larger than $\mathbf{B}_{0}$ early in the pulse.

The rapid and nonuniform heating of the target by the electron beam creates a magnetized plasma that carries the electron beam along as it expands.

\section{References}

1) M. Garcia, "On electromagnetic acceleration of material from a plate hit by a pulsed electron beam," Lawrence Livermore National Laboratory, UCRL-JC-130448, 16 April 1998.

4) Hydrodynamic calculations by P. Pincosy with CALE, Lawrence Livermore National Laboratory, April 1998.

\section{Figure legend}

1) Vector fields in the target plasma. This schematic shows how $\mathbf{j}_{0}, \mathbf{j}, \mathbf{B}, \nabla U$, and $\nabla \phi$ are related if the velocity $\mathbf{v}$ is outward. An axisymmetric arrangement is implied by the centerline symbol. The dotted lines indicate isotherms, while the thin solid lines radiating from the interior indicate equipotentials. The energy density $U$, pressure $p$, and conductivity $\sigma$ are also assumed to be largely uniform along isotherms. 


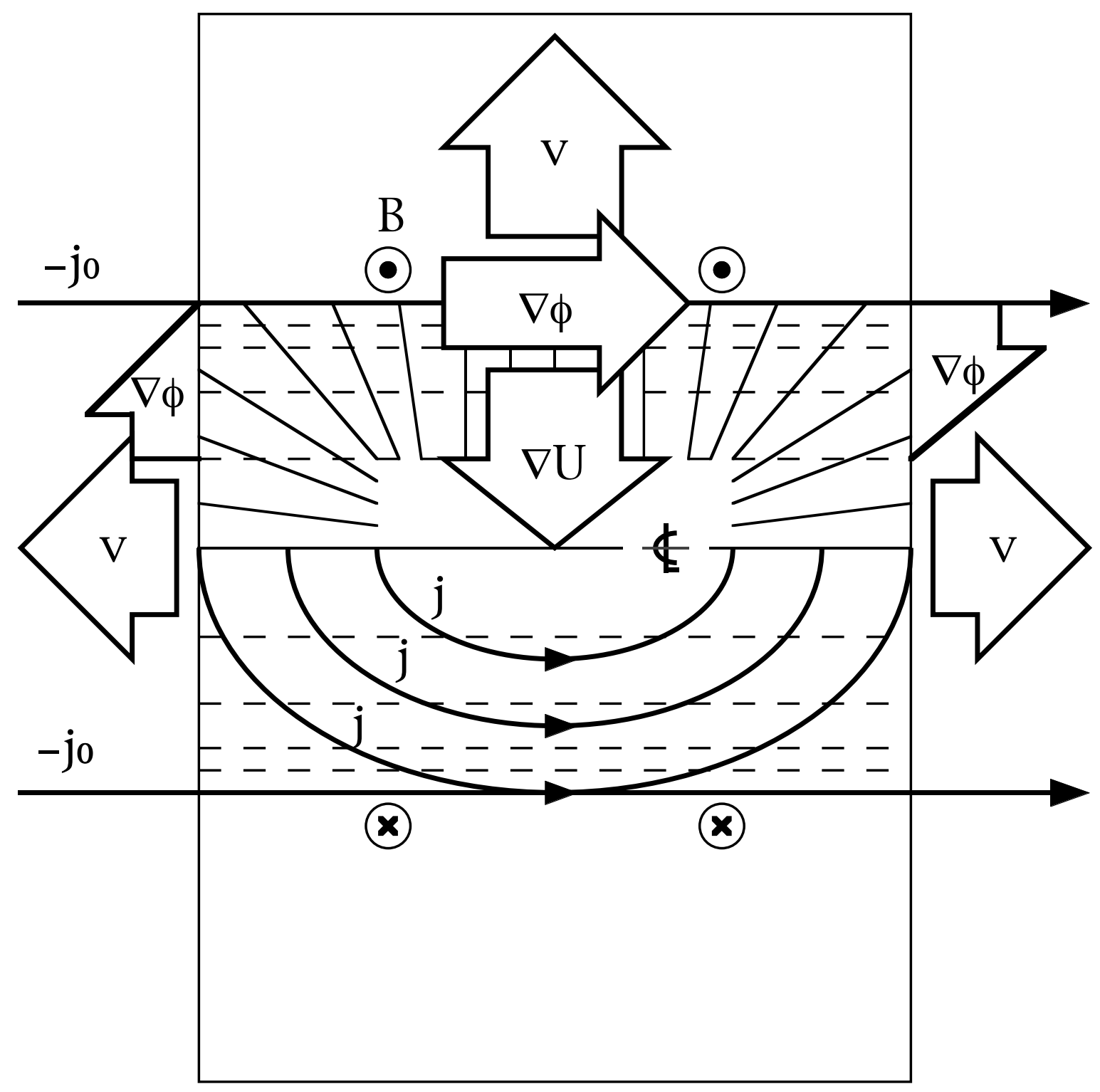

Figure 1. Vector fields in the target plasma 